Project 1022498

\title{
Importance of Mobile Genetic Elements and Conjugal Gene Transfer for Subsurface Microbial Community Adaptation to Biotransformation of Metals
}

\author{
Sorensen, Soren J. \\ University of Copenhagen
}

\section{RESULTS TO DATE: Annual Report 2005}

The overall goal of this project is to investigate the effect of mobile genetic elements and conjugal gene transfer on subsurface microbial community adaptation to mercury and chromium stress and biotransformation. Our studies focus on the interaction between the fate of these metals in the subsurface and the microbial community structure and activity.

Mercury-contaminated surface soil samples have been achieved from Lower East Fork Poplar Creek Floodplain at the FRC site. The mercury concentration was determined in the contaminated soils. The results showed that the concentrations decreased with depth. These findings were also reflected in the results of a mercury tolerance assay where the soil was exposed to increasing concentrations of mercury. The surface soil showed adaptation towards mercury since the respiration was reduced only slightly even at the highest mercury concentrations (100 ppm), whereas deeper soils (20?? and 40?? subsurface) showed minor adaptation. None of the soils showed any adaptation/resistance towards Chromate. Numbers of CFU?s resistant to mercury have been determined in the mercury contaminated and noncontaminated soils. The number of mercury resistant bacteria was higher in the contaminated soils. Furthermore, adaptation experiments have been performed including different microbiological analyses, e.g. plate counts, respiration, and BIOLOG $\mathrm{mt} 2$ and EcoMicroPlate readings. In these experiments it was found that the mercury-contaminated soils were more adapted to mercury than the non-contaminated soils. However, pre-exposure of the non-contaminated soils with mercury demonstrated that these soils also had an indigenous mercury resistant population - although at a much lower level than in the contaminated soils.

Isolation and characterization of hitherto uncultured bacteria of relevance for biotransformation of metals

The development of a method for cultivating hitherto uncultured soil bacteria, using solid membranes with soil bacteria, mounted on soil slurry (SSMS - Soil Slurry Membrane System) has been continued. The effect of incubating the membrane on the same soil slurry for an extended period relative to a setup where the slurry is repeatedly exchanged has been investigated. Analysis by PCR-DGGE of bacteria on the filters shows that the highest bacterial diversity is obtained if the soil slurry is replaced. Furthermore, we have been able to increase the culturability of the soil bacteria with a factor up to 180 . Approximately 800 bacterial CFU?s, originating from the SSMS setup, using sub-surface soil (18-22?? and 36-40?? depth) have been randomly picked and isolated. Based on their RAPD-pattern, 150 different isolates were identified by partial 16S rDNA sequencing. The sequences were grouped into 25 different profiles. Nine sequence profiles were found exclusively at 18-22? depth, and 8 were found exclusively at 36-40? depth. Eight sequence profiles were found at both depths. Continuous replacement of soil slurry below the membranes caused the appearance of new bacteria, as determined by $16 \mathrm{~S}$ rDNA sequencing. Representatives of Proteobacteria (?-, ?- and ?), Bacteroidetes, Firmicutes and Actinobacteria were all found among the SSMS isolated bacteria. BLAST searches showed that 9 of the 25 sequences had nearest neighbors of previously uncultured bacteria. These results show that the SSMS approach has a great potential for growing and isolating bacteria of hitherto unculturable species. Isolation of plasmids conferring resistance to mercury has been initiated. Of the 150 isolates having different RAPD-patterns, 15 were found to contain large plasmids and thus potentially conjugative. These will be further characterized and used in gene transfer experiments 
More than two hundred 16S rDNA clones, from mercury contaminated and non-contaminated soils, have been partially sequenced. Blast search shows that 16S rDNA clones show high similarity to the traditional ?big four? phyla; Proteobacteria, Firmicutes, Actinobacteria, Bacteroidetes, but also to members of the Acidobacteria, Nitrospira, Verrucomicrobia and Planctomycetes. The sequences are currently being analyzed. Low nutrient media containing a complex carbon source has previously been shown to increase culturability of soil bacteria. We are currently optimizing this media, for selective growth of mercury resistant soil bacteria. This media, along with traditional dilute solid media will be used to isolate mercury resistant bacteria. The isolates will be characterized with regards to their mercury resistance, with special focus towards merA diversity.

Horizontal gene transfer to ?non-culturable? sub-surface bacteria

Exogenous plasmid isolation, from both mercury contaminated and non-contaminated soil have been performed. Several conjugative plasmids have been isolated, and initially pooled into four different restriction fragment profiles. Their mercury reductase genes will be partially sequenced to determine their diversity. The plasmids will be examined by microarray analysis, to allow characterization of other potential heavy metal resistance genes, and to determine their repA profiles. Plasmid transfer efficiency to standard lab strains will be determined, and some plasmids will be tagged with GFP. We will use flow cytometry analysis to determine horizontal gene transfer occurrence in soil microcosms, and potentially use cell sorting and subsequent $16 \mathrm{~S}$ rDNA sequencing to determine transconjugant identity.

Significance of mobile genetic elements for microbial community adaptation to pollutants stress

We have designed a gene microarray containing 258 70-mer oligonucleotide targets; 100 to genes involved in resistance to heavy metals of interest in the field of bioremediation, such as $\mathrm{Hg}(\mathrm{II}), \mathrm{Pb}(\mathrm{II})$, and As(III), and 158 specific for replication genes (rep) of 86 transmissible broad host range plasmids belonging to 6 different incompatibility groups and 7 other distinct but currently uncharacterized phylogenetic groups. The microarray was optimized and rigorously tested with 28 exact-match control plasmids to assess the degree of cross-hybridization and the quality of the targets. The horizontal gene transfer array is currently used in the analysis of numerous plasmids that have been isolated previously from the subsurface at the FRC. We are planning to follow initial characterizations of FRC plasmids and their metal resistance genes with targeted sequencing of some plasmids. These experiments will identify the mechanisms by which metal resistance genes are spread in subsurface microbial communities. Such spread is critical to the ability of the indigenous community to sustain activities that are essential for bioremediation in mixed waste contaminated sites.

PCR analysis, targeting the mercuric reductase gene of Gram-negative bacteria, has shown that the mercury contaminated soils have higher levels of merA than the non-contaminated soils. Quantitative real time PCR (qrtPCR) has also been performed, and surprisingly, this cultivation-independent method seemed to underestimate the abundance of bacterial mercury resistance in soil. In order to obtain more reliable enumeration by qrtPCR, Firmicute-specific and Actinobacteria-specific merA primers have been designed. These primers, along with Proteobacteria-specific merA primers, will be used to evaluate merA abundance in soil. The merA diversity, obtained by PCR will be compared to merA diversity obtained by culturing, in order to evaluate the applicability of qrtPCR to analyse specific genes of interest in the environment.

DELIVERABLES: Manuscripts:

Barkay, T., and B.F. Smets. 2005. Horizontal gene flow in microbial communities: synthesis across scientific disciplines. ASM News 71:412-419

Smets B.F. and T. Barkay. 2005. Horizontal Gene Transfer Among Prokaryotes: Perspectives at a Crossroads of Scientific Disciplines. Nat. Rev. Microbiol. 3:675-678 Coombs, J.M., and T. Barkay. 2005. Horizontal gene transfer of metal homeostasis genes and its role in microbial communities of the 
deep terrestrial subsurface. Sixty-fifth Symposium of the Society of General Microbiology ?Microorganisms and Earth Systems - Advances in geomicrobiology?. pp. 109-129. Cambridge University Press, New York.

Coombs, J.M., and T. Barkay. Pathways in the evolution of metal homeostasis genes; evidence from the genomes of bacteria and archaea. Appl. Environ. Microbial. 71:7083-7091

Sorensen, S. J., Bailey, M., Hansen, L. H., Kroer, N. and S. Wuertz. 2005 Studying plasmid horizontal transfer in situ: A critical review. Nature Reviews Microbiology 3 (9): p700-710

Musovic, S., Oregaard, G., Kroer, N. \& Sorensen, S. J. Cultivation-independent detection of plasmid transfer to indigeous bacteria in Barley rhizosphere. Submitted Appl. Environ. Microbiol. (2005)

Johnsen, A.R. and Kroer, N. 2006 Assessment of Effects of Stress and other Environmental Factors on Plasmid Transfer by Direct Quantification of Discrete Transfer Events. FEMS Microb. Ecol. Under revision.

Rasmussen L.D., Binnerup S. and Kroer N. 2006. Cultivation by SSMS of hard to culture mercury resistant soil bacteria from two depths of a mercury-contaminated site (Oak Ridge, TN, USA). In prep.

Presentations

Sorensen S.J. 2005 Detection of horizontal gene transfer in natural environments by flow cytometry. Oral presentation at the ?IUMS meeting, July, San Francisco, USA. Invited.

Sorensen S.J. de Lipthay JR., Oregaard G.,Kroer, N., Rasmussen LD. 2005 Significance of Mobile Genetic Elements for Microbial Community Adaptation to Pollutant Stress. Poster presentation at the ?DOE-NABIR PI Meeting April 2005

Gunnar Oeregaard, Julia R. de Lipthay, Tamar Barkay, Soren J. Sorensen 2005 Cultivation-independent enumeration of mercury resistant bacteria from soil environments - pros and cons of quantitative real time PCR. Oral presentation at the ?A celebration of a Decade of Environmental Biotechnology Exchange Activities workshop, Brussels 17th October 2005. Invited.

Coombs, J., G. Oregaard, I. Torres, C.H. Black, P. Sobecky, and T. Barkay. A Functional Gene Microarray for the Detection of a Genomic Linkage Between Metal Resistance and inc/rep Genes on Broad Host Range Plasmids. 105th Annu. Meet. Am. Soc. Microbiol. Atlanta, June 5-9, 2005.

Barkay, T. Gene transfer in the soil environment. International Union of Microbiological Societies 2005. San Francisco. CA, July 23-28, 2005. Invited

Barkay, T. and J. Coombs. Horizontal gene transfer of metal homeostasis genes and its role in microbial communities of the deep terrestrial subsurface. Society of General Microbiology Symposium on ?Microorganisms in Earth Systems?, Keele, England, Sept. 12-15, 2005. Invited 